\title{
Population Risk Factors for COVID-19 Mortality in 93 Countries
}

\author{
Muhammad Jawad Hashim ${ }^{1,(1)}$, Ahmed R. Alsuwaidi², Gulfaraz Khan ${ }^{3, *}$ (D) \\ ${ }^{1}$ Department of Family Medicine, College of Medicine and Health Sciences (Tawam Hospital Campus), United Arab Emirates University, United Arab Emirates \\ ${ }^{2}$ Department of Pediatrics, College of Medicine and Health Sciences (Tawam Hospital Campus), United Arab Emirates University, United Arab Emirates \\ ${ }^{3}$ Department of Medical Microbiology and Immunology, College of Medicine and Health Sciences (Tawam Hospital Campus), \\ United Arab Emirates University, United Arab Emirates
}

\section{ARTICLE INFO}

\section{Article History}

Received 09 May 2020

Accepted 06 July 2020

\section{Keywords}

Novel coronavirus

SARS-CoV-2

COVID-19

epidemiology

risk factors

\begin{abstract}
Death rates due to COVID-19 pandemic vary considerably across regions and countries. Case Mortality Rates (CMR) per 100,000 population are more reliable than case-fatality rates per 100 test-positive cases, which are heavily dependent on the extent of viral case testing carried out in a country. We aimed to study the variations in CMR against population risk factors such as aging, underlying chronic diseases and social determinants such as poverty and overcrowding. Data on COVID-19 CMR in 93 countries was analyzed for associations with preexisting prevalence rates of eight diseases [asthma, lung cancer, Chronic Obstructive Pulmonary Disease (COPD), Alzheimer's Disease (AD), hypertension, ischemic heart disease, depression and diabetes], and six socio-demographic factors [Gross Domestic Product (GDP) per capita, unemployment, age over 65 years, urbanization, population density, and socio-demographic index]. These data were analyzed in three steps: correlation analysis, bivariate comparison of countries, and multivariate modelling. Bivariate analysis revealed that COVID-19 CMR were higher in countries that had high prevalence of population risk factors such as $\mathrm{AD}$, lung cancer, asthma and $\mathrm{COPD}$. On multivariate modeling however, $\mathrm{AD}, \mathrm{COPD}$, depression and higher GDP predicted increased death rates. Comorbid illnesses such as $\mathrm{AD}$ and lung diseases may be more influential than aging alone.
\end{abstract}

(C) 2020 The Authors. Published by Atlantis Press International B.V.

This is an open access article distributed under the CC BY-NC 4.0 license (http://creativecommons.org/licenses/by-nc/4.0/).

\section{INTRODUCTION}

The COVID-19 outbreak was first reported at the end of December 2019 from the city of Wuhan in China [1]. Within a short period of just 4 months, SARS-CoV-2, the coronavirus causing COVID-19, had spread to virtually all countries and territories worldwide [1]. In spite of implementing stringent measures such as travel restrictions, social distancing, lockdowns, and increased testing, many countries have struggled to control the spread and minimize the death toll [2]. At individual country level, the number of deaths and Case Fatality Rates (CFR) of confirmed cases vary enormously. This can be due to numerous reasons, including the extent of testing, the measures taken to mitigate the spread, healthcare access, underlying population demographics, socio-economic development and prevalence of comorbidities [3].

Moreover, different countries are at different stages of the COVID-19 pandemic and CFR can change depending on how each country responds to the epidemic. For example, UK, as of 25th June, had over 306,000 confirmed cases with over 43,000 deaths (CFR: 14.0\%). By contrast, Germany had 193,000 cases with just over 9000 death (CFR: $4.6 \%$ ). A recent study has indicated that SARS-CoV-2 has mutated into several different types which could have different virulence [4]. However, the order of magnitude

"Corresponding author.Email: g_khan@uaeu.ac.ae

Data availability statement: The authors confirm that the data supporting the findings

of this study are available within the article [and/or] its supplementary materials. difference in CFR noted between some countries cannot simply be explained by the variation in the virulence of the virus strains, since some of these countries have the same strain circulating [5]. Although the extent of testing is another obvious factor for these difference, it too does not explain all the differences.

The most common reported predictors of severe COVID-19 include age above 65 years and comorbidities such as cancer, asthma, hypertension and heart disease [6,7]. Thus, countries with larger older population and/or with higher burden of chronic diseases are expected to have the highest mortality rates. However, it is unclear how mortality rates interact with these reported predictors when examined at country population level by age and prevalence of comorbidities. We chose to analyze Case Mortality Rates (CMR) by country population, rather than by the number of test-positive cases (CFR), since the latter is dependent on the number of tests performed in each country and this varies enormously from country to country [8-10]. In this study, we aimed to examine CMR for 93 countries against eight diseases and six socio-demographic factors.

\section{MATERIALS AND METHODS}

\subsection{Study Design}

We analyzed COVID-19 pandemic data for variations in mortality rates across different countries. Data from Johns Hopkins University 
Coronavirus Resource Center (https://coronavirus.jhu.edu/data) were obtained on COVID-19 death rates per 100,000 population. These rates were used to classify countries into an elevated risk category. Prevalence data for selected diseases was retrieved from the Global Burden of Disease (GBD) dataset (http://ghdx.healthdata.org/gbd-results-tool). While this expansive dataset includes a wide range of health-related conditions including trauma and congenital birth defects, we limited our analysis to potential risk factors for COVID-19 mortality. These included asthma, Chronic Obstructive Pulmonary Disease (COPD), lung cancer, diabetes, depression, hypertension, Ischemic Heart Disease (IHD) and Alzheimer's Disease (AD). Socio-economic indicators were obtained from the World Bank data (https://databank.worldbank. org/home). Selected indicators were chosen to assess social risk factors for susceptibility. These included Gross Domestic Product (GDP) per capita in US dollars, unemployment rates (percent of workforce), urban population as a percent of total population, population density per square kilometer and population over age 65 years. An additional measure of social development, the Sociodemographic Index (SDI), from GBD was also analyzed. Expressed on a scale of 0 to 1 , SDI is the mean ranking of the national income per capita, educational attainment, and fertility rates. Data were merged with multiple error checking steps and redundancies.

Data were analyzed for 93 countries (primarily limited by availability of COVID-19 data). World Bank data from 2018 was used as it had a lower proportion of missing values compared with 2019. Missing values ranged from none (in the GBD data), to three countries (GDP) to 25 (unemployment rates) in the World Bank data. No data transformations were applied. The primary dependent (outcome) variable was COVID-19 death rates (CMR) per 100,000 population. We believe this metric is a more accurate measure than COVID-19 deaths per 100 confirmed cases (CFR) which is heavily dependent on the extent of viral case testing carried out in a country and the reliability of testing [8-10]. The data was analyzed in three steps: correlation analysis, bivariate comparison of countries, and finally, multivariate modelling.

\subsection{Statistical Analysis}

Statistical data analysis was conducted using Jamovi version 1.1.7 (The jamovi project, 2020, https://www.jamovi.org, Sydney, Australia). Generalized linear model (GAMLj module) was used to evaluate predictors for the dependent variable, CMR. Poisson regression was applied using the Log link function. Log likelihood ratio tests were used to evaluate the predictive performance of each covariate, while $R^{2}$ was maximized for overall model error reduction. Backward stepwise process was used to remove covariates with the greatest $p$-value at each iteration. Covariates were centered but not standardized. A fixed intercept was used. Two-way interaction terms were reviewed; however, none of them were statistically significant. Collinearity among predictor variables was assessed using a correlation matrix and tolerance in linear regression. Oneway ANOVA was used to compare the risk factors in elevated risk versus other countries. Mean differences in rates (including 95\% confidence intervals) were obtained as a measure of effect size. A correlation coefficient (cut-off) of $<0.25$ was considered as indicating lack of statistical association.

\section{RESULTS}

Globally, CMR was 10.4 COVID-19 deaths per 100,000 population, with highest rates in Belgium (84.9), Andorra (67.5), United Kingdom (64.3), Spain (60.6), Italy (57.3), Sweden (49.6), France (44.3) and the United States (36.7). By comparison, China's death rate stood at 0.33 and Iran at 11.8 per 100,000 (Supplementary Table S1).

\subsection{Correlation Analysis}

Country-wise correlations showed an association between total confirmed cases and total COVID-19 deaths $(r=0.93)$ and between confirmed cases and CMR $(r=0.29)$. SDI of the countries did not correlate with any of their COVID indicators such as confirmed cases and CMR $(r<0.25)$. Greater GDP per capita correlated with CMR $(r=0.39)$ but not with other COVID-19 variables such as number of cases. Having a relatively older population structure with a higher number of over 65 year old persons was associated with more confirmed cases $(r=0.36)$ and deaths $(r=0.33)$ but not with CMR. Social overcrowding as measured by population density and urbanization did not correlate with CMR $(r<0.25)$. From amongst the clinical risk factors, positive correlations with CMR included Alzheimer's disease $(r=0.36)$, lung cancer $(r=0.39)$, and weakly with asthma $(r=0.28)$ and COPD $(r=0.27)$. These correlations should be interpreted as preliminary exploratory analysis as confounding variables can mediate these findings.

\subsection{Grouped Comparison}

Countries were divided into two groups: those with CMR greater than 25 or more COVID-related deaths per 100,000 population, versus those with fewer deaths. The mean values of predictor variables were compared in these two groups using one-way ANOVA (Table 1). The results were consistent with correlation analysis, reaffirming greater prevalence of $\mathrm{AD}$ and respiratory illnesses in countries with high COVID-19 CMR. However, more conclusive inferences were derived using multivariate modeling.

\subsection{Multivariate Analysis}

With COVID-19 CMR per 100,000 population as the primary outcome (dependent) variable, multivariate modeling showed that certain risk factors were independent predictors $\left(R^{2}=0.35\right.$, log likelihood ratio tests, $p<0.05)$. These risk factors included Alzheimer's disease, COPD, depression and GDP per capita (Figure 1).

\section{DISCUSSION}

Key findings from our analysis highlight certain population risk factors that were associated with COVID-19 mortality. These included chronic respiratory diseases such as lung cancer, asthma and COPD and age-related illnesses like AD. A surprise finding from this study was the strong correlation between AD and COVID-19 death rates. Of the risk factors assessed, $\mathrm{AD}$ was a dominant and statistically significant risk factor, even on multivariate analysis (which controls 
Table 1 Comparison of countries grouped into elevated versus baseline COVID-19 deaths

\begin{tabular}{|c|c|c|c|c|}
\hline \multirow[b]{2}{*}{ Variable } & \multicolumn{2}{|c|}{ Mean values } & \multirow{2}{*}{$\begin{array}{l}\text { Difference } \\
\text { of means }\end{array}$} & \multirow{2}{*}{$\begin{array}{l}\text { One-way } \\
\text { ANOVA, } p^{\mathrm{b}}\end{array}$} \\
\hline & $\begin{array}{c}\text { Elevated risk } \\
\text { countries }(n=10)^{\mathrm{a}}\end{array}$ & $\begin{array}{l}\text { Other countries } \\
\qquad(n=83)\end{array}$ & & \\
\hline CMR & 53.6 & 5.2 & 48.4 & $<0.001$ \\
\hline CFR & 11.1 & 3.3 & 7.77 & $<0.001$ \\
\hline Cases & 346,000 & 62,000 & 284,000 & $<0.001$ \\
\hline \multicolumn{5}{|l|}{ Disease burden ${ }^{c}$} \\
\hline Alzheimer's disease & 1331 & 776 & 555 & $<0.001$ \\
\hline Lung cancer & 107 & 45 & 62 & $<0.001$ \\
\hline COPD & 6438 & 4561 & 1877 & $<0.001$ \\
\hline Asthma & 6142 & 4605 & 1537 & 0.016 \\
\hline Diabetes & 9720 & 8062 & 1658 & 0.086 \\
\hline IHD & 3005 & 2335 & 670 & 0.120 \\
\hline Depression & 2711 & 2216 & 495 & 0.012 \\
\hline Hypertension & 227 & 267 & -40 & 0.490 \\
\hline \multicolumn{5}{|l|}{ Socioeconomic factors $^{\mathrm{d}}$} \\
\hline GDP per capita & 48,804 & 19,282 & 29,522 & $<0.001$ \\
\hline Age $\geq 65$ years, millions & 12 & 6.1 & 5.9 & 0.380 \\
\hline Urbanization (\%) & 24 & 29 & -4.6 & 0.430 \\
\hline Population density & 188 & 246 & -58 & 0.839 \\
\hline Unemployment (\%) & 7.2 & 6.8 & 0.38 & 0.827 \\
\hline SDI & 0.69 & 0.72 & -0.03 & 0.543 \\
\hline
\end{tabular}

${ }^{a}$ Countries reporting more than 25 COVID-19 deaths per 100,000 population. ${ }^{b}$ Statistical significance on Tukey's post hoc testing with one-way ANOVA. 'Prevalence rates per 100,000 in 2017 from GBD study data set. ${ }^{\mathrm{d} D a t a}$ from the World Bank, 2018. CMR, COVID-19 deaths per 100,000 population; CFR, COVID-19 deaths per 100 confirmed cases.
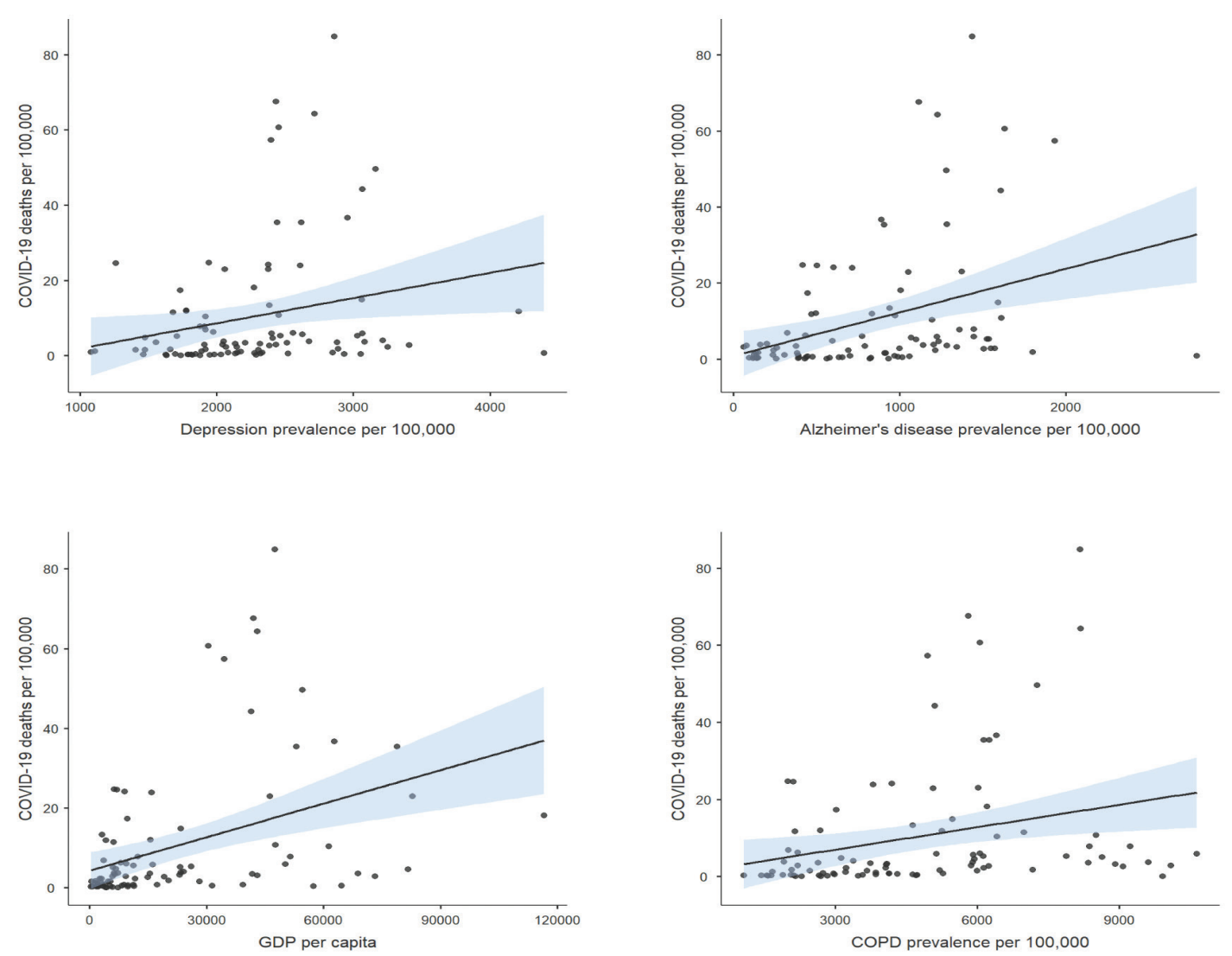

Figure 1 Population risk factors for COVID-19 deaths. $N=93$ countries. Prevalence data from Global Burden of Disease, 2017. 
for other risk factors including aging) [6,11]. Our findings support several recent studies that also point to a positive correlation between COVID-19 severity and neurological disorders, including $\mathrm{AD}$ [12-14]. The details of this association however, remain unknown. It has been reported that the virus has neurotropic characteristics, exploiting the angiotensin-converting enzyme 2 receptor to gain entry into cells of the central nervous system [15-17].

Another notable finding from this analysis was that countries with lower socio-economic development and social overcrowding were not associated with higher death rates. One of the major concerns has been the impact this pandemic will have on developing countries with poor infrastructure and healthcare systems. Our analysis appears to give some hope that low socio-economic status does not necessarily mean a guarantee of poor outcome for COVID-19. We must stress however, that our study focused on mortality and not on the risk of transmission. Interestingly, a previous study assessing infectious disease vulnerability scores also indicated that low income status does not necessarily correlate with high vulnerability to infections [18].

Studies have identified certain comorbidities such as age above 65 and hypertension as important risk factors for increased mortality $[6,19]$. Although this may be true when looking at crude figures, analyzing the risk of mortality in multivariate modeling shows only weak correlation. Thus, countries with a higher percentage of older people may witness a higher burden of deaths $[19,20]$ but this is not because older people are specifically at higher risk of developing severe disease compared with younger individuals in the population. Other clinical risk factors such as co-morbid illnesses, Alzheimer's dementia and chronic lung diseases (COPD) may be more influential.

In a recent systematic review, hypertension and diabetes were reported as leading risk factors for COVID-19 severity [21]. These were followed by cardiovascular diseases and respiratory conditions. It is pertinent to note that data from hospital-based series should not be used to make public health decisions elsewhere as these associations are context dependent. Each medical center receives a unique set of patients depending on referral patterns, pre-hospital care and clinical expertise.

\subsection{Limitations}

Limitations of our study include the use of secondary data, estimation techniques used by the data sources, accuracy of data estimates and the potential of ecological bias due to confounding by countrylevel analysis. We sought to minimize bias and systematic errors by removing outliers and not relying on case-fatality rates. It is worth mentioning that observational studies on clinical cases suffer from similar confounding effects that are difficult to remove even by adjustment or stratification. Definitive proof via randomized exposure to the causative virus would be unethical. Hence, observational data provide the best available source for public health and clinical decision making.

\section{CONCLUSION}

The current COVID-19 pandemic has exposed some of the weaknesses in the public healthcare systems and lack of preparedness for dealing with infectious disease outbreaks [22]. Emerging and re-emerging infections, particularly due to viruses are not new and COVID-19 is certainly not going to be the last. Previous pandemics such as the 1918 influenza pandemic, famously known as the 'Spanish flu' and the two subsequent influenza pandemics of 1957 and 1968 resulted in millions of deaths $[23,24]$. To limit the devastating impact such pandemics can have on human health and healthcare systems, our only options are to be better prepared for such events. One essential component for this preparedness is to have established policies for future pandemics. Early identification, testing, contact tracing, and isolation are fundamental principles of public health that have to be implemented. Determining which groups in a population are at increased risk of severe disease can help to better manage the limited resources and stretched healthcare systems in such situations. Moreover, any planning for lifting the existing lockdown measures should take into consideration these vulnerable groups. This is also true for prioritizing recipients for candidate vaccines against COVID-19.

\section{CONFLICTS OF INTEREST}

The authors declare they have no conflicts of interest.

\section{AUTHORS' CONTRIBUTION}

MJH Design of the protocol, acquisition of data, data analysis/ interpretation, preparing first draft and critically reviewing the paper, giving approval for the final version to be published. ARA Critically reviewing the paper, giving approval for the final version to be published. GK Principal investigator and corresponding author, conception/design of the protocol, data analysis/interpretation, preparing first draft and critically reviewing the paper, giving approval for the final version to be published.

\section{FUNDING}

This research received no external funding.

\section{ACKNOWLEDGMENTS}

We would like to thank the sources of original data including Johns Hopkins University, Institute of Health Metrics (University of Washington, Seattle) and the World Bank.

\section{SUPPLEMENTARY MATERIAL}

Supplementary data related to this article can be found at https:// doi.org/10.2991/jegh.k.200721.001.

\section{REFERENCES}

[1] Zhu N, Zhang D, Wang W, Li X, Yang B, Song J, et al. A novel coronavirus from patients with pneumonia in China, 2019. N Engl J Med 2020;382;727-33.

[2] Qian GQ, Chen XQ, Lv DF, Ma AHY, Wang LP, Yang NB, et al. Duration of SARS-CoV-2 viral shedding during COVID-19 infection. Infect Dis 2020;52;511-12. 
[3] Kucharski AJ, Russell TW, Diamond C, Liu Y, Edmunds J, Funk S, et al. Early dynamics of transmission and control of COVID-19: a mathematical modelling study. Lancet Infect Dis 2020;20;553-8.

[4] Forster P, Forster L, Renfrew C, Forster M. Phylogenetic network analysis of SARS-CoV-2 genomes. Proc Natl Acad Sci U S A 2020;117;9241-3.

[5] Hadfield J, Megill C, Bell SM, Huddleston J, Potter B, Callender C, et al. Nextstrain: real-time tracking of pathogen evolution. Bioinformatics 2018;34;4121-3.

[6] Epidemiology Working Group for NCIP Epidemic Response, Chinese Center for Disease Control and Prevention. [The epidemiological characteristics of an outbreak of 2019 novel coronavirus diseases (COVID-19) in China]. Zhonghua Liu Xing Bing Xue Za Zhi 2020;41;145-51 [Article in Chinese].

[7] World Health Organization (WHO). Report of the WHOChina joint mission on coronavirus disease 2019 (COVID-19) [Internet]. Geneva, Switzerland: World Health Organization; 2020. Available from: https://www.who.int/publications-detail/ report-of-the-who-china-joint-mission-on-coronavirus-disease2019-(covid-19) [cited April 14, 2020].

[8] Onder G, Rezza G, Brusaferro S. Case-fatality rate and characteristics of patients dying in relation to COVID-19 in Italy. JAMA 2020;323;1775-6.

[9] Liang C, Cao J, Liu Z, Ge F, Cang J, Miao C, et al. Positive RT-PCR test results after consecutively negative results in patients with COVID-19. Infect Dis 2020;52;517-19.

[10] Hase R, Kurita T, Muranaka E, Sasazawa H, Mito H, Yano Y. A case of imported COVID-19 diagnosed by PCR-positive lower respiratory specimen but with PCR-negative throat swabs. Infect Dis 2020;52;423-6.

[11] Zhou F, Yu T, Du R, Fan G, Liu Y, Liu Z, et al. Clinical course and risk factors for mortality of adult inpatients with COVID-19 in Wuhan, China: a retrospective cohort study. Lancet 2020; 395; 1054-62.

[12] Kuo CL, Pilling LC, Atkins JL, Masoli JAH, Delgado J, Kuchel GA, et al. APOE e4 genotype predicts severe COVID-19 in the UK Biobank community cohort. J Gerontol A Biol Sci Med Sci 2020;glaa131.
[13] Mao L, Jin H, Wang M, Hu Y, Chen S, He Q, et al. Neurologic manifestations of hospitalized patients with coronavirus disease 2019 in Wuhan, China. JAMA Neurol 2020;77;683-90.

[14] Docherty AB, Harrison EM, Green CA, Hardwick HE, Pius R, Norman L, et al. Features of 20133 UK patients in hospital with covid-19 using the ISARIC WHO clinical characterisation protocol: prospective observational cohort study. BMJ 2020;369;m1985.

[15] Lu R, Zhao X, Li J, Niu P, Yang B, Wu H, et al. Genomic characterisation and epidemiology of 2019 novel coronavirus: implications for virus origins and receptor binding. Lancet 2020;395;565-74.

[16] Li YC, Bai WZ, Hashikawa T. The neuroinvasive potential of SARS-CoV2 may play a role in the respiratory failure of COVID-19 patients. J Med Virol 2020;92;552-5.

[17] Baig AM, Khaleeq A, Ali U, Syeda H. Evidence of the COVID19 virus targeting the CNS: tissue distribution, host-virus interaction, and proposed neurotropic mechanisms. ACS Chem Neurosci 2020;11;995-8.

[18] Moore M, Gelfeld B, Okunogbe A, Paul C. Identifying future disease hot spots: infectious disease vulnerability index. Rand Health Q 2017;6;5.

[19] Wynants L, Van Calster B, Collins GS, Riley RD, Heinze G, Schuit E, et al. Prediction models for diagnosis and prognosis of covid-19: systematic review and critical appraisal. BMJ 2020;369;m1328.

[20] Jordan RE, Adab P, Cheng KK. Covid-19: risk factors for severe disease and death. BMJ 2020;368;m1198.

[21] Yang J, Zheng Y, Gou X, Pu K, Chen Z, Guo Q, et al. Prevalence of comorbidities and its effects in patients infected with SARS-CoV-2: a systematic review and meta-analysis. Int J Infect Dis 2020;94;91-5.

[22] Anderson M, Mckee M, Mossialos E. Covid-19 exposes weaknesses in European response to outbreaks. BMJ 2020;368;m1075.

[23] Viboud C, Simonsen L, Fuentes R, Flores J, Miller MA, Chowell G. Global mortality impact of the 1957-1959 influenza pandemic. J Infect Dis 2016;213;738-45.

[24] Taubenberger JK, Morens DM. 1918 Influenza: the mother of all pandemics. Emerg Infect Dis 2006;12;15-22. 